\title{
Visual Analytics for Electronic Intelligence: Challenges and Opportunities
}

\author{
Alma Cantu* ${ }^{* \dagger}$, Olivier Grisvard*†, Thierry Duval* \\ * IMT Atlantique, Lab-STICC, Univ. Bretagne Loire, \\ Technopôle Brest-Iroise, 29238 Brest, France \\ \{alma.cantu, olivier.grisvard, thierry.duval\}@ imt-atlantique.fr \\ $\dagger$ Thales Airborne Systems, \\ 10 avenue de la 1ère DFL, 29238 Brest, France
}

\begin{abstract}
In this paper, we present the field of Electronic Intelligence (ELINT) and the issues that it raises for visual analytics. ELINT aggregates many of the actual issues that visual analytics face such as huge amounts of data, complex data, complex tasks, missing data and unreliable data. This aggregation of specificities makes ELINT a domain raising many visualization issues. This paper identifies the challenges of ELINT by describing its specificities and identifies the opportunities that ELINT raises for the visualization domain.
\end{abstract}

Keywords-Visual analytic application, Electronic intelligence, Multidimensional data, Temporal data

\section{INTRODUCTION}

The domain of Electronic Intelligence (ELINT), as many others, has to deal with an increasing amount of data. This domain also requires the processing of very complex tasks. Thus, it deeply relies on automatic processing. However, existing techniques for automatic analysis reach their limits due to the nature of the data to process (incomplete and noisy). As such, a part of these data still has to be processed manually, which is in the scope of visual analytics. In this paper, we identify the challenges and opportunities of ELINT regarding visualization. To that purpose, we first describe the ELINT challenges, answering the what, why and how questions thanks to the knowledge acquired during interviews of ELINT operators. Then, we propose some visual analytics opportunities specific to the ELINT analysis that highlight the strengths and weaknesses of the ELINT field in terms of visualization.

\section{ELINT CHALLENGES}

Radar detection consists in sending an electromagnetic emission that is reflected by objects and then intercepted back by the radar. The way the signal is distorted when reflected gives information on the objects such as distance or size. To increase the detection, the radar signals have complex characteristics that make their signature unique. Such emissions can be intercepted by other sensors, namely electronic support measures. Electronic warfare exploits the interception of radar emissions to identify their origin, characterize their threat level and enable the appropriate reaction in case of conflict, or conversely, to prevent an opponent from doing the same. However, the identification of a radar requires having a precise knowledge of the existing radar signatures. Gathering this knowledge is an activity generally performed by dedicated military forces and called ELINT. It consists in capturing and analyzing as much emissions as possible in order to characterize their signatures and as such fill the reference database used in electronic warfare.

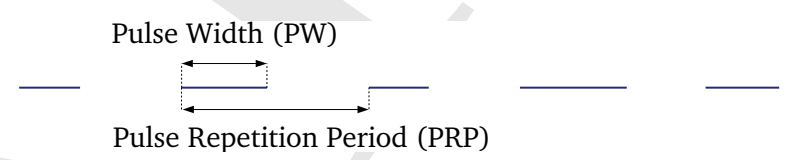

Fig. 1. Illustration of a radar signal signature (for representation purposes, PW and PRP values appear to have the same scale, but in fact PW is insignificant compared to PRP).

\section{A. ELINT data type}

Radar signals consist in series of pulses of a certain duration, spaced in time (see Figure 1). These pulses are emitted on a specific frequency (such as radio emissions) and with a specific power, named level. Thus, pulse width (PW), pulse repetition period (PRP), frequency and level are the four attributes that characterize a pulse. These attributes may vary from one pulse to another in a same signal, but always according to a recurrent pattern in order to make possible the interpretation by the emitter. This pattern is what the ELINT operators look for, but not directly what the ELINT data set contains. The rawest form of data used in ELINT consists of samples of frequency and level measured through time. If a series of temporally adjacent samples has a high enough level and coherent frequencies, it is automatically characterized as a shaped pulse (SP). Because of the huge amount of data, SPs are synthesized as rectangular pulses $(R P s)$, recording the average level and average frequency of SPs (see Figure 2). These inner variations are often radarspecific but taking them into account increases the complexity of the task out of proportion with the operational needs. In most cases, considering four varying attributes is discriminant enough to identify a signal. As we have seen so far, the ELINT data that correspond to shaped pulses have singular frequency, level, $P W$ and time (named time of arrival, TOA) values. In order to access the PRP, they also contain the computed delta of TOA (DTOA). ELINT data sets may also contain 
other attributes such as the direction of arrival (DOA). These additional attributes help to distinguish several radar signals as they ensure constant values specific to each signal and therefore easy to cluster. Concerning the data volume, as the capacities of current Radars are confidential, we cannot detail this matter. But if we refer to studies made a few years ago [7] and if we consider the increase of data storage capacities, we can state that the current data volume is huge.

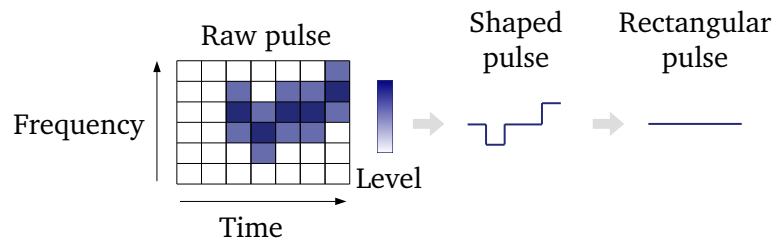

Fig. 2. Illustration of the pulse acquisition process.

According to Shneiderman's definition [12], the ELINT data correspond to multidimensional data, because the cardinality of the attributes exceeds three, and to temporal data, because they contain the time as attribute. This allows us to specify the data description using a characterization specific to multidimensional and temporal data. Detailing the multidimensional aspect requires characterizing the properties of each attribute. The ELINT data attributes are TOA, DTOA, PW, level and frequency. These attributes are all of quantitative type and are ordered in a sequential direction [9, Chapter 2]. Regarding time data characterization, Aigner et al. proposes a classification based on three aspects: linear time vs. cyclic time, time points vs. time intervals and ordered time vs. branching time vs. time with multiple perspectives [1, Chapter 3]. Despite the ELINT data contain a strong cyclic aspect due to inner patterns, they cannot be considered as falling in the category of cyclic time and as such correspond to linear time. The cyclic aspect concerns the data and not the time value. Concerning the point vs. interval aspect, as an impulsion has a length, named PW, the ELINT data may be considered as time intervals. However, as PW values are insignificant compared to the time scale, PW is rather considered as a full part attribute and the data as time points. Finally, the ELINT data time values have an important quantitative aspect from a single point of view and as such are neither ordinal nor with multiple perspectives and correspond to Aigner et al. definition of branching time. Regarding the data volume, if we consider the human perception limit and the current devices used to visualize ELINT data, the amount of data is such that its rendering exceeds the human perception capacity [6].

\section{B. ELINT goals}

To formally describe the different ELINT goals we use the tasks taxonomy highlighted by Purchase et al. [14], [8], [10]. We use this taxonomy despite many more recent studies [2], [11] because it is detailed enough to describe the ELINT field and simple enough to be applied intelligibly to such a complex field. The activity of an ELINT operator is to identify the characteristics of a radar signal in order to determine its signature and as such make its identification possible in the future. These characteristics are the pattern values (PW, PRP and frequency), physical indicators (level variations) and context indicators (DOA, position of the emitter). As such, the main tasks of an ELINT operator is to locate patterns and identify characteristics. However these tasks are not directly accessible due to several constraints that require performing additional tasks. In this subsection we detail these additional tasks and their origin.

1) Tasks brought by data complexity: The ELINT activity is performed on multidimensional data. Without a representation encoding the five main attributes at the same time, representations must be duplicated and as such, tools are required to associate different representations of a same data. The complexity is also brought by the length of the patterns. Extracting a pattern that contains more than four elements is not a pre-attentive process [5] and as such requires a specific comparing task.

2) Tasks brought by data quantity: We have stated previously that representing entire ELINT datasets raises perception issues. The main part of these data is automatically processed. However, the small percentage remaining still corresponds to large quantities. Moreover, some analyses require to be processed knowing the whole context, which involves the use of the whole dataset. Dealing with such amounts of data does not require specific tasks but impacts the analysis by requiring more time, more operators or by constraining the tasks with scale considerations.

3) Tasks brought by noise: The electromagnetic waves are reflected by the environment such that the radar signals are often surrounded by duplicates and other electromagnetic sources such as radio emissions. All these extra emissions create an ambient noise easily detectable because of the weakness of their signals (level value). However, some meaningful pulses can also have low level values. To handle this issue, ELINT operators filter low level values to detect patterns and analyze the filtered noise after the pattern characteristics are established.

4) Tasks brought by missing data: Sometimes the recording sensors miss some pulses. These missing pulses raise two issues. First, a missing pulse means a missing part of the pattern. Secondly, missing pulses raise PRP identification issues. Accessing the PRP value relies on a computed value, the DTOA. Yet if a pulse went missing, the time delta between the pulses around it will be at least twice the real one so that the DTOA will not represent accurately the PRP (see Figure 3). Because of this false value, the pulse before the missing pulse may be considered as noise leading to another missing part of the pattern. If the pattern is detectable despite missing pulses, it requires to identify the characteristics of the missing pulse from another recurrence and insert artificial pulses correcting the DTOA value. If not, handling these issues requires to identify a recurrent DTOA value and access pulses with DTOA values equal to a multiple of this DTOA.

5) Tasks brought by mixed data: It may occur that sensors record two radar signals at the same time. This mix of signals 

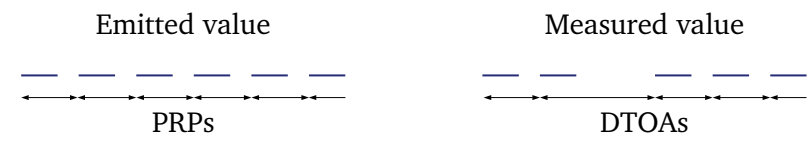

Fig. 3. Illustration of the differences that a signal degradation can make between emitted and measured signals.

raises two issues. First, it mixes patterns such that the ELINT operators have to first differentiate the radar signals before trying to identify their patterns. Performing this differentiation requires clustering the radar signals. Luckily, one of the radar attributes (mainly the frequency) is often constant, such that in most of the cases identifying a single radar signal means to categorize pulses with similar values. However, the exception where all values are varying happens and there are no established solutions to handle it. Secondly, mixed signals impact the exactness of the DTOA value regarding the PRP value as illustrated figure 4. As such, to access the correct DTOA also requires to first differentiate the signals.
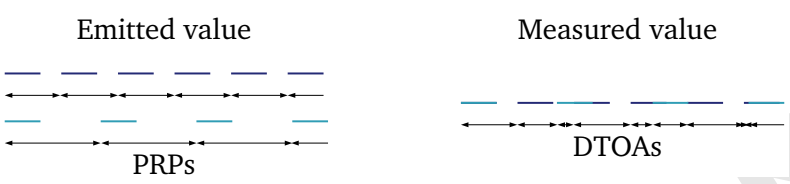

Fig. 4. Illustration of the impact of mixed signals on DTOA.

6) Tasks brought by unreliable data: In order to record the most accurate frequency value, there are multiple frequency sensors, each recording a specific frequency range. If a radar signal has a frequency close to the junction of two frequency range sensors, the same pulse may be recorded several times, one by each sensor (see Figure 5). Such pulses will be present twice in the dataset with a more or less accurate frequency value. This issue is recognizable by close frequency clusters and by tiny DTOA values (the pulses are received almost at the same time). To handle this duplication and choose the pulses with the most accurate frequency value, the operators correlate the frequency range to the level values, rank the frequency clusters according to their level and filter on the frequency range that have the highest level values (the highest level corresponds to the best signal strength, so to the most accurate).

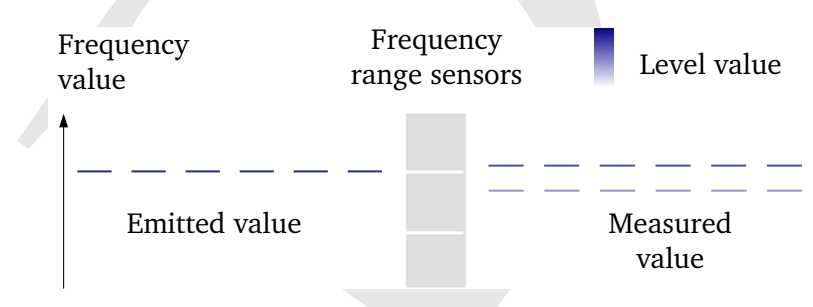

Fig. 5. Illustration of the listening of a radar signal on two different frequency range sensors.

\section{ELINT context of use}

ELINT analysis can be performed under many human factors related constraints (on board of an aircraft or a ship), or under less stressed conditions (in ground centers). Regarding the user experience, the military turnover does not always allow the operators to acquire the high level of expertise necessary to the analysis of the complex cases. Regarding the platform, the only constraint concerns the hardware performance (GPU/CPU) which may be limited, especially on board. As such, it may be impossible to deploy visualization solutions requiring too high performance of the hardware.

\section{Synthesis}

ELINT deals with a huge amount of temporal and multidimensional data structured through the relations of their attributes. These data are used to perform a large variety of complex tasks such as they cover the entire taxonomy described by Purchase et al. [10]: identify, categorize, locate features, rank, compare, distinguish (filter), associate and correlate. Moreover, these tasks are performed in varying contexts, including constrained environments and users with low expertise.

\section{ELINT REPRESENTATIONS}

In this section we present the most common representations currently used to perform the ELINT activity. We highlight their weaknesses and propose opportunities to solve some of their issues.
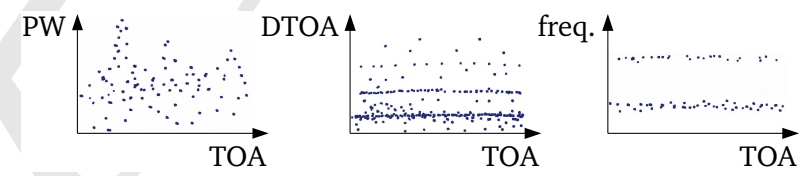

Fig. 6. Illustration of a current ELINT representation.

\section{A. Drawbacks of existing tools}

The most common representation used for ELINT analysis consists of several 2D charts (see Figure 6). On these charts, each pulse is represented by an object and positioned according to two of its attributes, one is often the time. According to Mackinlay [4] the position strongly allows us to perceive the quantitative, ordinal and nominal properties of data. As such, using position to represent data that have these three properties, as ELINT data do, enables us to access the three of them. However, the ELINT activity mainly requires accessing the nominal aspect of the data in order to distinguish or associate them. As there is no need to get the quantitative aspect of the data, the position provides too much insight and may cause a cognitive overload. In addition, due to the quantity of data processed by ELINT, these representations are impacted by occlusion effects. This allows us to access contextual knowledge but does not make it possible to focus on details. The quantity of data also induces scalability issues. For instance, two frequency clusters can have mean values that differ from $10^{-3} \mathrm{MHz}$ and be contained in a record 
where frequency values vary from 0 to $10^{4} \mathrm{MHz}$. Such a scaling difference makes it impossible for human eyes to detect these clusters on desktop screens. More than disabling spontaneous detection, if we consider that the user is aware of this scale issue, it requires to zoom and to unzoom along the entire frequency axes, which can be tedious. Furthermore, the ELINT analysis processes many multidimensional tasks such as detecting patterns and identifying their characteristics. Using 2D charts does not allow us to correlate more than two attributes at the same time, which impacts the performance of multidimensional tasks. To conclude, despite that current ELINT representations accurately render the content of the data, the quantity and complexity of the data raise issues that these representations do not handle.

\section{B. Opportunities}

In this part, we present some proposals about how to handle the previous issues for ELINT.

1) Color hue instead of position for attributes of a pattern:

Hypothesis: Using the color hue instead of the position for encoding attributes of a pattern decreases the user's cognitive load and facilitates the pattern detection for ELINT.

Rationale: According to Mackinlay [4], the color hue, contrarily to the position, does not permit a good perception of the quantitative property of data. We have established previously that this quantitative aspect is superfluous for ELINT activity such that disabling it may reduce the cognitive load of the users. Concerning pattern detection, according to Ware [13], the color is the most important factor when it comes to pre-attentive processing. Color immediately pops out from a set of other features, which facilitates the detection of the recurrent values that compose a pattern. Following the Gestalt theory [3], when elements are placed close to each other they tend to be perceived as a group, and contrarily when elements are placed without proximity they tend to be perceived as separate shapes. As values in patterns are not necessarily close values, representing pattern attributes by the position will separate them spatially. If the color is used instead of the position for attributes of the pattern, the elements of a same pattern will be restrained to close positions, which may make them perceived as a group, making the pattern detection easier.

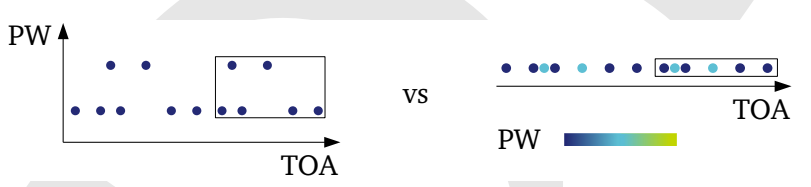

Fig. 7. Illustration of the use of position in $2 \mathrm{D}$ to encode PW and TOA versus the use of color and position in one dimension.

2) Ordinal distribution in time:

Hypothesis: Distributing data in an ordinal manner does not impact the ELINT analysis but avoids occlusion.

Rationale: The time value and its quantitative aspect are not necessary to ELINT analysis as all the required information is contained in the DTOA values. As such, distributing data in an ordinal manner according to time does not impact the ELINT analysis and makes it possible to avoid occlusion.

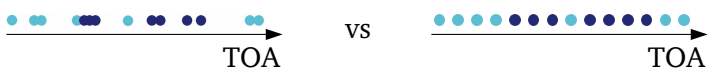

Fig. 8. Illustration of ELINT data displayed in time, respectively in a quantitative and in an ordinal manner.

\section{3) Parallel coordinates:}

Hypothesis: Using parallel coordinates instead of 2D charts facilitates the pattern detection and the identification of multidimensional data characteristics.

Rationale: In previous paragraphs, we have seen that using the position facilitates the association between elements that have close values, but since pulses that follow each other in a pattern have different values and since recursive pulses are separated in time, none of them can be associated. If we remove the time axis by representing data on one dimension, the recursive pulses will benefit from the association effect brought by the position. Moreover, the use of connections between different representations of a data facilitates the correlation and the identification of multidimensional data characteristics.

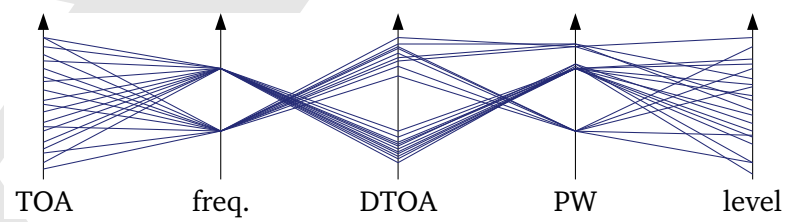

Fig. 9. Illustration of parallel coordinates for ELINT.

\section{CONCLUSION}

The use of visual analytics techniques for ELINT is a peculiar challenge. The amount of data to be represented is huge and increasing. ELINT data are highly complex since they contain more than five attributes, including time. These data, corresponding to radar signals, contain patterns, singularities and missing data that the ELINT operators have to identify through various tasks. Existing visual analytics techniques provide some solutions, but the amount of noise, the working scale, the missing data and the number of attributes raise issues that existing techniques cannot handle. We have highlighted some recommendations to build new representations better matching the ELINT context. In further work, we aim to validate the proposed recommendations through experimentations and build visual analytics solutions based on these recommendations.

\section{REFERENCES}

[1] Wolfgang Aigner, Silvia Miksch, Heidrun Schumann, and Christian Tominski. Visualization of time-oriented data. Springer-Verlag London, 2011. 
[2] Matthew Brehmer and Tamara Munzner. A multi-level typology of abstract visualization tasks. IEEE Transactions on Visualization and Computer Graphics, 19(12):2376-2385, 2013.

[3] Vicki Bruce, Patrick R Green, and Mark A Georgeson. Visual perception: Physiology, psychology, \& ecology. Psychology Press, 2003.

[4] Stuart K Card, Jock D Mackinlay, and Ben Shneiderman. Readings in information visualization: using vision to think. Morgan Kaufmann, 1999.

[5] Stanislas Dehaene. The number sense: How the mind creates mathematics. OUP USA, 2011.

[6] T. J. Jankun-Kelly, Tim Dwyer, Danny Holten, Christophe Hurter, Martin Nöllenburg, Chris Weaver, and Kai Xu. Scalability considerations for multivariate graph visualization. In Andreas Kerren, Helen C. Purchase, and Matthew O. Ward, editors, Multivariate Network Visualization, pages 207-235. Springer International Publishing, Cham, 2014.

[7] Jukka-Pekka Kauppi, Kalle Martikainen, and Ulla Ruotsalainen. Hierarchical classification of dynamically varying radar pulse repetition interval modulation patterns. Neural Networks, 23(10):1226-1237, 2010.

[8] Peter R. Keller and Mary Keller. Visual Cues: Practical Data Visualization. IEEE Computer Society, Los Alamitos, USA, 1993.

[9] Tamara Munzner. Visualization Analysis and Design. CRC Press, 2014.

[10] Helen C. Purchase, Natalia Andrienko, T. J. Jankun-Kelly, and Matthew Ward. Theoretical Foundations of Information Visualization, pages 4664. Springer Berlin Heidelberg, Berlin, Heidelberg, 2008.

[11] Hans-Jörg Schulz, Thomas Nocke, Magnus Heitzler, and Heidrun Schumann. A design space of visualization tasks. IEEE Transactions on Visualization and Computer Graphics, 19(12):2366-2375, 2013.

[12] Ben Shneiderman. The eyes have it: A task by data type taxonomy for information visualizations. In Visual Languages, 1996. Proceedings., IEEE Symposium on, pages 336-343. IEEE, 1996.

[13] Colin Ware. Information visualization: perception for design. Elsevier, 2012.

[14] S. Wehrend and C. Lewis. A problem-oriented classification of visualization techniques. Proceedings of the First IEEE Conference on Visualization: Visualization'90, pages 139 - 143, 469, 1990. 